International Journal of Pure and Applied Mathematics

Volume 87 No. 2 2013, 349-354

ISSN: $1311-8080$ (printed version); ISSN: 1314-3395 (on-line version)

url: http://www.ijpam.eu

doi: http://dx.doi.org/10.12732/ijpam.v87i2.15

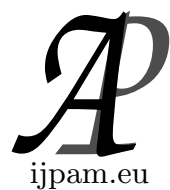

\title{
AUTHENTICATED MULTIPLE KEY DISTRIBUTION USING SIMPLE CONTINUED FRACTION
}

\author{
S. Srinivasan ${ }^{1}$, P. Muralikrishna ${ }^{2 \S}$, N. Chandramowliswaran ${ }^{3}$ \\ ${ }^{1,2}$ School of Advanced Sciences \\ VIT University \\ Vellore, 632014, Tamilnadu. INDIA \\ ${ }^{3}$ Visiting Faculty \\ Indian Institute of Management Indore \\ Indore, 453 331, INDIA
}

\begin{abstract}
In resent years the security of operations taking place over a computer network become very important. It is necessary to protect such actions against bad users who may try to misuse the system (e.g. steal credit card numbers, read personal mail, or impersonate other users.) Many protocols and schemes were designed to solve problem of this type. Threshold cryptography is a novel cryptographic technique sharing secret among members. It divides a secret key into multiple shares by a cryptographic operation. In this paper, we proposed a key distribution algorithm based on Simple Continued Fraction. The goal of our algorithm is to divide a secret $S$ into $\ell$ pieces $s_{1}+s_{2}+\cdots+s_{\ell}$ such that, $\ell$ pieces are necessary to reconstruct $S$, but any $m<\ell$ pieces give no information about $S$.
\end{abstract}

AMS Subject Classification: 94A60, 94A62

Key Words: simple continued fraction, shares, RSA prime

\section{Introduction}

Secret sharing (also called secret splitting) refers to method for distributing a

Received: June 15, 2013

(c) 2013 Academic Publications, Ltd.

$\S$ Correspondence author url: www.acadpubl.eu 
secret amongst a group of participants, each of whom is allocated a share of the secret. The secret can be reconstructed only when a sufficient number, of possibly different types, of shares are combined together; individual shares are of no use on their own.

Secret sharing was invented independently by Adi Shamir [3] and George Blakley [1] in 1979. Secret sharing schemes are ideal for storing information that is highly sensitive and highly important. Examples include: encryption keys, missile launch codes and numbered bank accounts. Each of these pieces of information must be kept highly confidential, as their exposure could be disastrous, however, it is also critical that they not be lost. Traditional methods for encryption are ill-suited for simultaneously achieving high levels of confidentiality and reliability. This is because when storing the encryption key, one must choose between keeping a single copy of the key in one location for maximum secrecy, or keeping multiple copies of the key in different locations for greater reliability. Increasing reliability of the key by storing multiple copies lowers confidentiality by creating additional attack vectors; there are more opportunities for a copy to fall into the wrong hands. Secret sharing schemes address this problem, and allow arbitrarily high levels of confidentiality and reliability to be achieved.

A secure secret sharing scheme distributes shares so that anyone with fewer than $t$ shares has no extra information about the secret than someone with 0 shares.

Consider for example the secret sharing scheme in which the secret phrase security is divided into the shares $s e------,--c u----,----r i--$, and $------t y$. A person with 0 shares knows only that the password consists of eight letters. He would have to guess the password from $26^{8}=208$ billion possible combinations. A person with one share, however, would have to guess only the six letters, from $26^{6}=308$ million combinations, and so on as more persons collude. Consequently this system is not a secure secret sharing scheme, because a player with fewer than $t$ secret-shares is able to reduce the problem of obtaining the inner secret without first needing to obtain all of the necessary shares.

\section{Proposed Algorithms}

\section{Construction of the Non-Homogeneous Equation}

Definition An expression of the form $C_{0}+\frac{1}{C_{1}+} \frac{1}{C_{2}+} \frac{1}{C_{3}+} \ldots \frac{1}{+C_{n}} \ldots$ where $C_{j}$ : 
$j \geq 1$ are positive integers and $C_{0}$ is a non-negative integer is called a Simple Continued Fraction (SCF).

- Define $\frac{P_{1}}{Q_{1}}=\frac{C_{0}}{1}$

$$
\begin{aligned}
& \frac{P_{2}}{Q_{2}}=C_{0}+\frac{1}{C_{1}}=\frac{C_{0} C_{1}+1}{C_{1}} \\
& \frac{P_{3}}{Q_{3}}=C_{0}+\frac{1}{C_{1}+\frac{1}{C_{2}}}=\frac{C_{0}\left[C_{1} C_{2}+1\right]+C_{2}}{C_{1} C_{2}+1}
\end{aligned}
$$

- In general, we have

$$
\begin{aligned}
& \frac{P_{n+1}}{Q_{n+1}}=C_{0}+\frac{1}{C_{1}+\frac{1}{C_{2}+} \frac{1}{C_{3}+} \cdots \frac{1}{+C_{n}}} \\
& =C_{0}+\frac{1}{C_{1}+\frac{1}{C_{2}+\frac{1}{C_{3}+\cdots+\frac{1}{C_{n-1}+\frac{1}{C_{n}}}}}}
\end{aligned}
$$

then, $\frac{P_{n+1}}{Q_{n+1}}$ is called $(n+1)^{t h}$ convergent of $S C F$

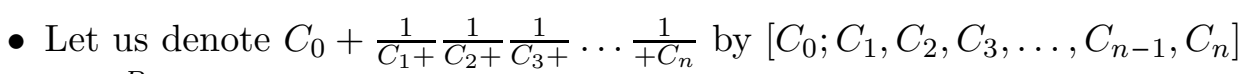
i.e., $\frac{P_{n+1}}{Q_{n+1}}=\left[C_{0} ; C_{1}, C_{2}, C_{3}, \ldots, C_{n-1}, C_{n}\right]$

- Alternatively, we can represent $S C F$ as the product of special $2 \times 2$ matrices over non-negative integers

$$
\left[\begin{array}{cc}
C_{0} & 1 \\
1 & 0
\end{array}\right]\left[\begin{array}{cc}
C_{1} & 1 \\
1 & 0
\end{array}\right] \quad \ldots \quad\left[\begin{array}{cc}
C_{n} & 1 \\
1 & 0
\end{array}\right]=\left[\begin{array}{cc}
P_{n+1} & P_{n} \\
Q_{n+1} & Q_{n}
\end{array}\right]
$$

- Taking determinant on both sides of (4), we get $(-1)^{n+1}=P_{n+1} Q_{n}-Q_{n+1} P_{n}$ for all $n \geq 1$

- Taking transpose of (4), we get

$$
\left[\begin{array}{cc}
C_{n} & 1 \\
1 & 0
\end{array}\right]\left[\begin{array}{cc}
C_{n-1} & 1 \\
1 & 0
\end{array}\right] \quad \ldots \quad\left[\begin{array}{cc}
C_{0} & 1 \\
1 & 0
\end{array}\right]=\left[\begin{array}{cc}
P_{n+1} & Q_{n+1} \\
P_{n} & Q_{n}
\end{array}\right]
$$

- Let $R_{1}, R_{2}$ be two given very large odd primes with $R_{1}<R_{2}$

- Represent $\frac{R_{1}}{R_{2}}$ as a $S C F$

$$
\frac{R_{1}}{R_{2}}=C_{0}+\frac{1}{C_{1}+} \frac{1}{C_{2}+} \frac{1}{C_{3}+} \ldots \frac{1}{+C_{n}}=\left[C_{0} ; C_{1}, C_{2}, C_{3}, \ldots, C_{n-1}, C_{n}\right]
$$

- Consider the symmetric continue fraction of length $2 n+2$

$$
\frac{P_{2 n+2}}{Q_{2 n+2}}=\left[C_{n} ; C_{n-1}, C_{n-2}, \ldots, C_{1}, C_{0}, C_{0} ; C_{1}, C_{2}, C_{3}, \ldots, C_{n-1}, C_{n}\right]
$$
where $\frac{P_{i}}{Q_{i}}$ be the $i^{\text {th }}$ convergent of (7)

- $\frac{P_{n+1}}{Q_{n+1}}=\left[C_{n} ; C_{n-1}, C_{n-2}, \ldots, C_{1}, C_{0}\right]$

$$
\begin{aligned}
& \frac{P_{n}}{Q_{n}}=\left[C_{n} ; C_{n-1}, C_{n-2}, \ldots, C_{1}\right] \\
& \frac{P_{n+1}}{P_{n}}=\left[C_{0} ; C_{1}, C_{2}, C_{3}, \ldots, C_{n-1}, C_{n}\right] \\
& \frac{Q_{n+1}}{Q_{n}}=\left[C_{0} ; C_{1}, C_{2}, C_{3}, \ldots, C_{n-1}\right]
\end{aligned}
$$


- Therefore
$\left[\begin{array}{cc}C_{n} & 1 \\ 1 & 0\end{array}\right]\left[\begin{array}{cc}C_{n-1} & 1 \\ 1 & 0\end{array}\right] \ldots$
$=\left[\begin{array}{ll}P_{2 n+2} & P_{2 n+1} \\ Q_{2 n+2} & Q_{2 n+1}\end{array}\right]=A$
$\left[\begin{array}{cc}C_{1} & 1 \\ 1 & 0\end{array}\right]\left[\begin{array}{cc}C_{0} & 1 \\ 1 & 0\end{array}\right]\left[\begin{array}{cc}C_{0} & 1 \\ 1 & 0\end{array}\right]\left[\begin{array}{cc}C_{1} & 1 \\ 1 & 0\end{array}\right] \quad \ldots \quad\left[\begin{array}{cc}C_{n} & 1 \\ 1 & 0\end{array}\right]$

- Clearely, $\operatorname{det}(A)=(-1)^{2 n+2}=1$

- Consider

$\left[\begin{array}{cc}C_{n} & 1 \\ 1 & 0\end{array}\right]\left[\begin{array}{cc}C_{n-1} & 1 \\ 1 & 0\end{array}\right] \ldots \quad\left[\begin{array}{cc}C_{1} & 1 \\ 1 & 0\end{array}\right]\left[\begin{array}{cc}C_{0} & 1 \\ 1 & 0\end{array}\right]\left[\begin{array}{cc}C_{0} & 1 \\ 1 & 0\end{array}\right]\left[\begin{array}{cc}C_{1} & 1 \\ 1 & 0\end{array}\right] \quad \ldots \quad\left[\begin{array}{cc}C_{n} & 1 \\ 1 & 0\end{array}\right]$

$=\left[C_{n} ; C_{n-1}, \ldots, C_{1}, C_{0}, C_{0} ; C_{1}, C_{2}, \ldots, C_{n-1} C_{n}\right]$

$=\left[\begin{array}{cc}P_{n+1} & P_{n} \\ Q_{n_{1}} & Q_{n}\end{array}\right]\left[\begin{array}{cc}P_{n+1} & Q_{n+1} \\ P_{n} & Q_{n}\end{array}\right]$

$=\left[\begin{array}{cc}P_{n+1}^{2}+P_{n}^{2} & P_{n+1} Q_{n+1}+P_{n} Q_{n} \\ P_{n+1} Q_{n+1}+P_{n} Q_{n} & Q_{n+1}^{2}+Q_{n}^{2}\end{array}\right]$

$=\left[\begin{array}{ll}P_{2 n+2} & P_{2 n+1} \\ Q_{2 n+2} & Q_{2 n+1}\end{array}\right]$

- $P_{2 n+2} Q_{2 n+1}-P_{2 n+1} Q_{2 n+2}=1$

$\left(P_{n+1}^{2}+P_{n}^{2}\right) Q_{2 n+1}=P_{2 n+1}^{2}+1$

$\left(R_{1}^{2}+R_{2}^{2}\right) Y=X^{2}+1$

where $P_{n+1}=R_{1}, P_{n}=R_{2}, P_{2 n+1}=X$ and $Q_{2 n+1}=Y$

Here, we proposed an algorithm and its actual implementation. Our primary contribution is that, in our algorithm we discussed the key sharing by using two prime numbers and a non-homogeneous equation with two variables. The share holders can verify the validity of their shares only after reconstruction of the secret and any share holders fewer than $\ell$, can not reconstruct the secret.

\section{Algorithm for Encryption}

- Choose two secret very large odd primes $R_{1}, R_{2}$ with $R_{1}>R_{2}$

- Construct $x^{2}+1=\left(R_{1}^{2}+R_{2}^{2}\right) y$

- Select two large RSA secret odd primes $p$ and $q$

- Define $N=p q$ then $\phi(N)=(p-1)(q-1)$ where $\phi(N)$ is Euler phi function

- Select a secret exponent $e$ such that $(e, \phi(N))=1$ where $1<e<\phi(N)$

- For this $e$, there is a unique $d$ such that $e d \equiv 1(\bmod \phi(N))$ 


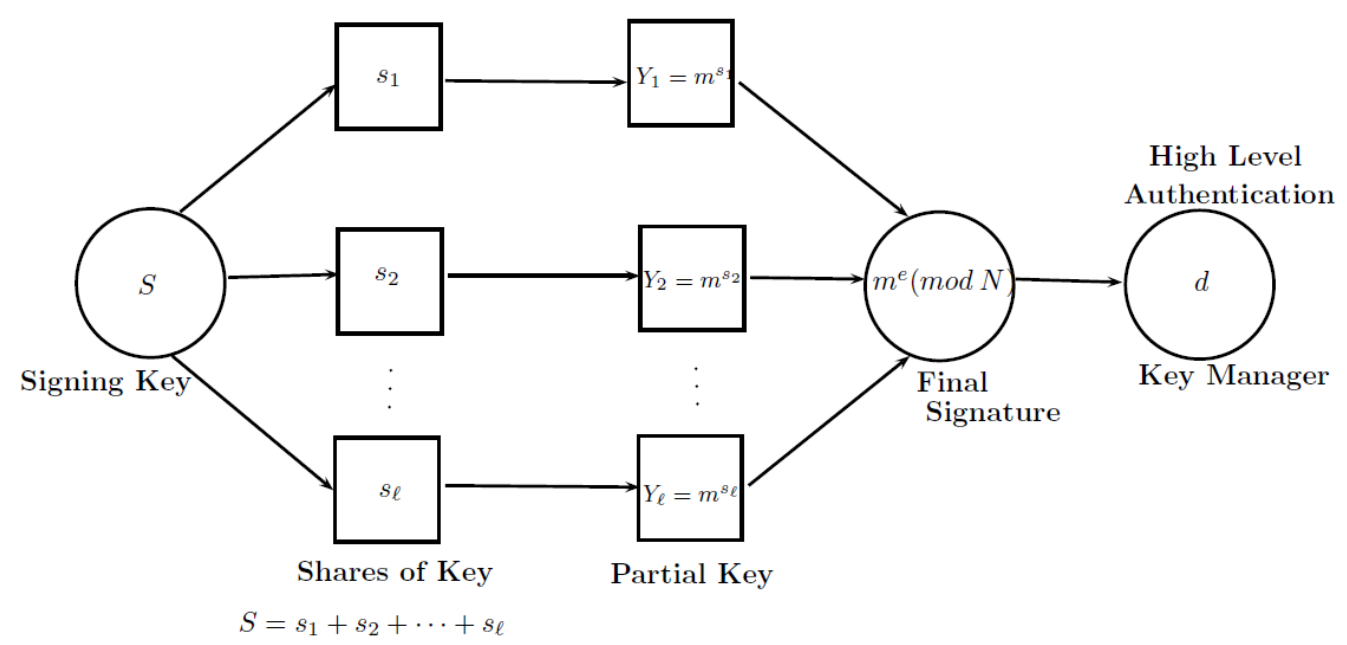

- consider $a=\left(R_{1}^{2}+R_{2}^{2}\right)(y+d)-(x+\phi(N))^{2}$

$$
\begin{aligned}
& a=\left(R_{1}^{2}+R_{2}^{2}\right) y-x^{2}+\left(R_{1}^{2}+R_{2}^{2}\right) d-[\phi(N)]^{2}-2 x \phi(N) \\
& \quad=1+\left(R_{1}^{2}+R_{2}^{2}\right) d-[\phi(N)]^{2}-2 x \phi(N) \\
& a \equiv\left(1+\left(R_{1}^{2}+R_{2}^{2}\right) d\right)(\bmod \phi(N)) \\
& a e \equiv\left(e+\left(R_{1}^{2}+R_{2}^{2}\right)\right)(\bmod \phi(N)) \\
& \quad S \equiv e(\bmod \phi(N)) \text { where } S=a e-\left(R_{1}^{2}+R_{2}^{2}\right)
\end{aligned}
$$

- Here we define $S$ is the exponent secret

- Represent the secret message $m$ in the interval $[0, N-1]$ with $\operatorname{gcd}(m, N)=1$

\section{Algorithm for Key Sharing}

- Consider the $\ell$ exponent secret partition (share holders)

$S=s_{1}+s_{2}+\cdots+s_{\ell}$

Define $Y_{i} \equiv m^{s_{i}}(\bmod N)$ for $1 \leq i \leq \ell$

$$
\begin{aligned}
\prod_{1}^{\ell} Y_{i} & \equiv m^{S}(\bmod N) \\
& \equiv m^{s_{1}+s_{2}+\cdots+s_{\ell}}(\bmod N) \\
& \equiv m^{s_{1}} m^{s_{2}} \ldots m^{s_{\ell}}(\bmod N)
\end{aligned}
$$

- For $\ell$ secret share holders we can distribute $\ell$ key's such as $Y_{1}, Y_{2}, \ldots, Y_{\ell}$

- Sharing scheme follows from the given equations:

$$
\prod_{1}^{\ell} Y_{i} \equiv m^{S}(\bmod N)
$$




$$
\begin{aligned}
& \equiv m^{k \phi(N)+e}(\bmod N) \\
& \equiv m^{k \phi(N)} m^{e}(\bmod N) \\
& \equiv\left[m^{\phi(N)}\right]^{k} m^{e}(\bmod N) \\
\prod_{1}^{\ell} Y_{i} & \equiv m^{e}(\bmod N)
\end{aligned}
$$

- High Level Authentication Key manager is d

$$
\begin{aligned}
& \left(\prod_{1}^{\ell} Y_{i}\right)^{d} \equiv\left(m^{e}\right)^{d}(\bmod N) \\
& \left(\prod_{1}^{\ell} Y_{i}\right)^{d} \equiv \bmod (\bmod N)
\end{aligned}
$$

\section{Conclusion}

This paper dealt with a secret key sharing mechanism on $\ell$ share holders. According to the algorithm, the secret can be well shared with feasible sense, it may have some performance impact when the number of share holders is accordingly increased. Our approach to secret sharing is opened a number of avenues for future work. These include research into finding schemes that will remove the restrictions on the size of $\ell$. These schemes make it possible to store secret information in a network.

\section{References}

[1] Adi Shamir, How to share a secret, Communications of the ACM, 22, No. 11 (1979), 612-613.

[2] A. Beimel, Secret-sharing schemes: a survey, In: Proceedings of the Third International Conference on Coding and Cryptology, Berlin, Heidelberg, 2011, Springer-Verlag, IWCC'11, 11-46.

[3] G.R. Blakley, Safeguarding cryptographic keys, Proceedings of the National Computer Conference, 48 (1979), 313-317.

[4] P. Muralikrishna, S. Srinivasan, N. Chandramowliswaran, Secure schemes for secret sharing and key distribution using Pell's equation, International Journal of Pure and Applied Mathematics, 85, No. 5, 33-937, doi: 10.12732/ijpam.v85i5.11.

[5] Ivan Niven, Herbert S. Zuckerman, Hugh L. Montgomery, An Introduction to the Theory of Numbers, John Wiley.

[6] Tom M. Apostol, Introduction to Analytic Number Theory, Springer. 